\title{
Multi-term multiple prediction using separated reflections and diffractions combined with curvelet-based subtraction
}

\author{
D.J. Verschuur*, Delft University of Technology, Deli Wang, Jilin University and F.J. Herrmann, EOS-UBC
}

\section{Summary}

The surface-related multiple elimination (SRME) method has proven to be successful on a large number of data cases. Most of the applications are still 2D, as the full 3D implementation is still expensive and under development. However, the earth is a $3 \mathrm{D}$ medium, such that $3 \mathrm{D}$ effects are difficult to avoid. Most of the 3D effects come from diffractive structures, whereas the specular reflections normally have less of a 3D behavior. By separating the seismic data in a specular reflecting and a diffractive part, multiple prediction can be carried out with these different subsets of the input data, resulting in several categories of predicted multiples. Because each category of predicted multiples can be subtracted from the input data with different adaptation filters, a more flexible SRME procedure is obtained. Based on some initial results from a Gulf of Mexico dataset, the potential of this approach is investigated.

\section{Introduction}

The surface-related multiple elimination (SRME) method (Berkhout, 1982; Verschuur et al., 1992; Berkhout and Verschuur, 1997; Weglein et al., 1997) has developed itself as one of the standard multiple removal tools in today's seismic data processing sequences. During the last decade, the interest in multiple elimination techniques in a full 3D implementation has grown significantly. Although quite some advancements have been made, still full 3D surfacerelated multiple removal is an expensive process, which is still under development. Therefore, there is still room for extending the 2D implementation of SRME. Furthermore, it can be argued that the proposed process can also help the full $3 \mathrm{D}$ implementation, given the fact that none of the current 3D SRME processes yields perfectly predicted multiples.

In this paper a strategy is proposed for data from environments in which the $3 \mathrm{D}$ effects are limited. In those situations, the 3D effects are most noticeable in the diffractive part of the seismic response. The specular reflections have travel paths in which the out-of-plane effects are limited, but as soon as diffractive structures are present in the earth, strong out-of-plane behavior can arise. When the predicted multiples are subjected to a subtraction process from the input data, the mis-predicted multiple diffractions will limit the overall subtraction output, despite the fact that the reflective part of the multiples is reasonably predicted.
Kabir (2003) already suggested a procedure to handle reflections and diffractions separately by a filtering process applied to the predicted multiples. In this paper we take this approach one step further. It is argued that the separation of reflections and diffractions, such as described by Khaidukov et al. (2004) or Taner et al. (2006), can be applied already before the prediction phase, such that in the end four categories of multiples can be predicted. Each of those categories can be matched to the input data with its own matching filter, such that the overall subtraction process becomes more flexible.

\section{Surface-related multiple prediction for specular reflections and diffractions}

In Berkhout and Verschuur (1997) it has been proposed to rewrite the surface-related multiple removal scheme of Verschuur et al. (1992) as an iterative procedure:

$$
\mathbf{P}_{0}{ }^{(\mathrm{i}+1)}=\mathbf{P}-\mathbf{P}_{0}^{(\mathrm{i})} \mathbf{A} \mathbf{P},
$$

$\mathbf{P}_{0}{ }^{(i)}$ representing the pre-stack data containing the estimated primaries and internal multiples in iteration $i, \mathbf{P}$ being the total data (primaries and all multiples) and $\mathbf{A}$ represents the so-called surface operator:

$$
\mathbf{A}=\mathbf{S}^{-1} \mathbf{R}^{\wedge} \mathbf{D}^{-1},
$$

in which the inverse source and receiver properties are combined with the reflectivity at the free surface from below. The notation is taken from Berkhout (1982). Each column of a data matrix, e.g. P, contains a wave field (or a shot record) for one frequency. Thus, the full matrix contains a pre-stack data volume for one frequency component.

In practice, the directivity effects are often negelected or taken into account in a separate preprocessing step, such that matrix can be written as a frequency dependent scalar $\mathrm{A}(\omega)$ (Verschuur and Berkhout, 1997).

Thus the prediction of the surface-related multiples can be written as:

$$
\mathbf{M}^{(\mathrm{i}+1)}=\mathbf{P}_{0}^{(\mathrm{i})} \mathbf{P},
$$

After which $\mathrm{A}(\omega)$ is determined in an adaptive subtraction process, often based on minimum energy in the primaries:

$$
\mathbf{P}_{0}{ }^{(i+1)}=\mathbf{P}-\mathrm{A}(\omega) \mathbf{M}^{(\mathrm{i}+1)} .
$$




\section{Separate prediction of specular and diffracted multiples}

The multiple prediction process, as given by Equation (3), describes a combination of the two datasets: the estimated primaries and the total input data. This means that if we can subdivide either of the two datasets into different parts, that add up to the original data, the prediction can still be carried out. For the purpose of handling 3D effects in a better way, it is proposed to split the data into a specular and a diffraction part. Taner et al. (2006) described a method to make this separation. It is based on the fact that for the specular reflections Snell's law will be obeyed. If the reflectors in the earth are reasonably horizontally oriented, this means that for the specular reflections there is a relationship between the incidence angle of the wave field at the source side and the arrival angle at the receiver side: they should be more or less opposite in sign. Therefore, a transformation of the seismic data to the ray parameter domain along both the source and the receiver coordinate enables us to select the specular part of the data. The rest is defined as the diffractions. Of course, the process for the actual separation between reflections and diffraction is a bit subjective and the separation filter has to be chosen with care, to avoid filtering artifacts.

Thus, if the total data $\mathbf{P}$ can be separated in a reflection part $\mathbf{P}_{\mathrm{r}}$ and in a diffraction part $\mathbf{P}_{\mathrm{d}}$, and the same is done for the estimate of the primaries, the multiple prediction scheme of Equation (3) can be rewritten as:

$$
\mathbf{M}^{(\mathrm{i}+1)}=\left[\mathbf{P}_{0, \mathrm{r}}{ }^{(\mathrm{i})}+\mathbf{P}_{0, \mathrm{~d}}{ }^{(\mathrm{i})}\right]\left[\mathbf{P}_{\mathrm{r}}+\mathbf{P}_{\mathrm{d}}\right],
$$

meaning that we will get four different categories of predicted multiples:

$$
\begin{aligned}
& \mathbf{M}_{\mathrm{rr}}{ }^{(i+1)}=\mathbf{P}_{0, \mathrm{r}}{ }^{(\mathrm{i})} \mathbf{P}_{\mathrm{r}}, \\
& \mathbf{M}_{\mathrm{rd}}{ }^{(\mathrm{i}+1)}=\mathbf{P}_{0, \mathrm{r}}{ }^{(\mathrm{i})} \mathbf{P}_{\mathrm{d}}, \\
& \mathbf{M}_{\mathrm{dr}}{ }^{(\mathrm{i}+1)}=\mathbf{P}_{0, \mathrm{~d}}{ }^{(\mathrm{i})} \mathbf{P}_{\mathrm{r}}, \\
& \mathbf{M}_{\mathrm{dd}}{ }^{(\mathrm{i}+1)}=\mathbf{P}_{0, \mathrm{~d}}{ }^{(\mathrm{i})} \mathbf{P}_{\mathrm{d}},
\end{aligned}
$$

Each of these multiples can be subtracted with their own adaptation process. In this way, diffractions can be subtracted with a filter that differs from the one used for the specular part of the multiples. 3D effects can thus be accomodated for.

\section{Curvelet-based subtraction}

It is expected that the least-squares subtraction method, as proposed by Verschuur and Berkhout (1997) will not yield good result, as the diffracted parts will still contain errors that cannot be resolved by a simple time-domain convolution filter. Therefore, a different adaptation process is considered, which can handle larger prediction errors.
Herrmann and Verschuur $(2004,2005)$ have proposed a methodology based on the curvelet-transform. This process involves a transformation of the predicted multiples and the input data to the curvelet domain, in which a thresholding process is carried out. Under the constraint of a sparse representation of both estimated primaries and multiples, the curvelet coefficients where the predicted multiples show large amplitudes are suppressed for the total data. With this thresholding process, small errors in localization, frequency content and dip can be accommodated.

Furthermore, by using four different types of prediction terms, the thresholding process can be cascaded for each type of predicted multiples.

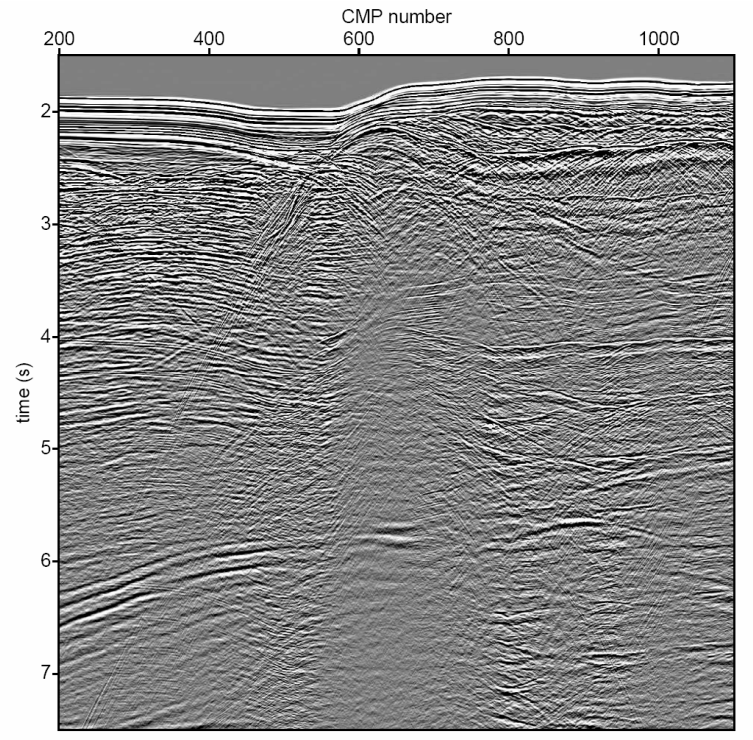

Figure 1: Stack section with multiples for a Gulf of Mexico dataset (courtesy WesternGeco). Note that this is a deep-water situation, the first order multiples being recorded from 3.4 seconds.

\section{Field data example: Gulf of Mexico}

An initial example of this approach is demonstrated for a 2D line from the Gulf of Mexico, from the Mississippi Canyon (Courtesy WesternGeco). In Figure 1 a stack with multiples is displayed for part of this line. Note that this area is characterized by lateral variations in the water depth and that also diffractive structures can be well recognized. First, the process as described by Taner et al. (2006) is applied to the input shot records to separate the specular part from the diffractive part. For this example it involves the following processing steps:

- Transformation of each shot record to the $\tau$-p domain;

- Resort the data for constant ray-parameter; 


\section{Separate prediction of specular and diffracted multiples}

- Extract the horizontal components by a spatial alphatrimmed median filter along the source coordinate for each ray-parameter;

- Resort the result into shot gathers;

- Inverse transformation from the $\tau-\mathrm{p}$ to the $\mathrm{x}$-t domain.

The result after these steps is the specular part of the input data. By subtracting this from the original data, the diffractive part is obtained.
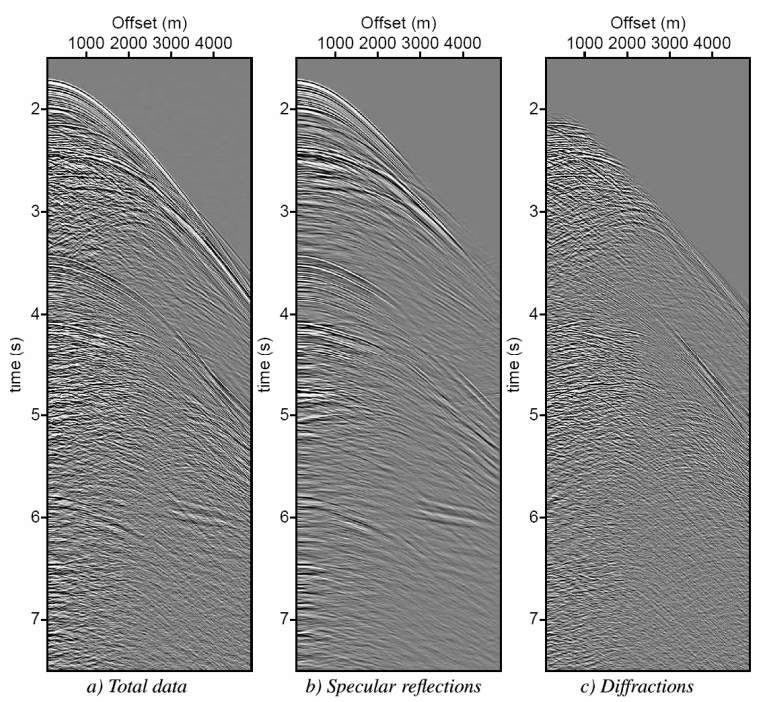

Figure 2: a) Shot record with all multiples. b) Estimated specular reflection part of the shot record. c) Estimated diffractive part of this shot record (i.e. difference of $a$ and $b$ ).

In Figure 2 the result of applying this process is shown for one shot record, which is located in the middle of the section in Figure 1. Note that although one shot record is shown, it is a multi-shot process. In Figure 2a the input shot record with all multiples is shown. Figure $2 b$ shows the extracted specular part of the input data and Figure 2c displays the difference between the input and the specular part, which are the diffractions. Actually, some additional muting has been applied to the diffraction part of the data to remove the steep residual primaries at the large offsets, which are slightly aliased. Note that Fomel et al. (2006) also used this dataset for the purpose of separately imaging the diffractions, in order to extract velocity information from the diffractions via a post-stack migration process.

Next, the prediction of four categories of predicted multiples are carried out with the data being separated in reflections and diffractions, according to equation (6a-d). Note again that we consider here the first iteration, where the input data is also used as multiple prediction operator.
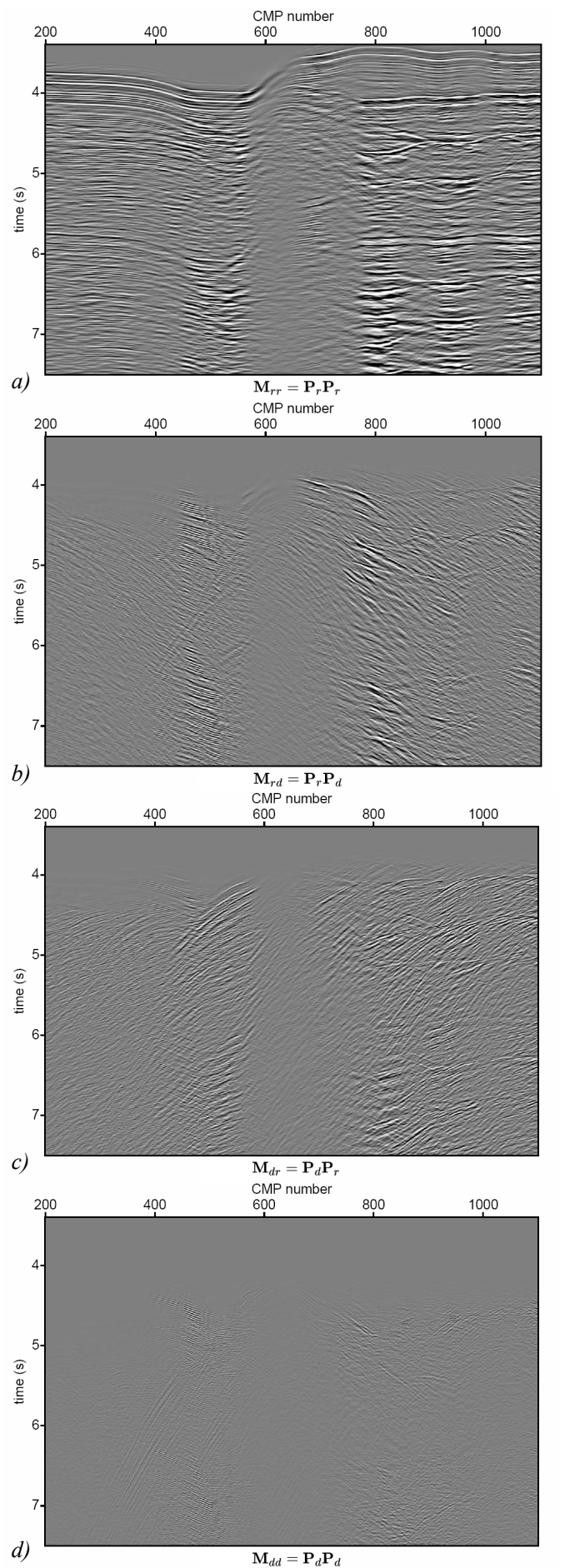

Figure 3: Stacks made from the predicted multiples in four subcategories, after separating the data into a specular and diffractive part. Note that the diffracted multiples are in the last three plots. 


\section{Separate prediction of specular and diffracted multiples}

Thus, four different versions of surface multiples are constructed. For display purposes, each category of predicted multiples is subjected to a stacking procedure. The results are displayed in Figure 3a-d. Note that the specular part of the predicted multiples is laterally smooth, as is expected (see Figure 3a). Also observe that the two combinations of diffractions and reflections (Figure $3 \mathrm{~b}$ and Figure 3c) show the more complex part of the multiples. In addition, we observe that both parts have opposite dips for the events. Finally, Figure 3d shows the result of the diffractive part of the operator convolved with itself, showing the most complex part of the multiples. This latter part gives the weakest contribution, as expected.

Finally, the predicted multiples are subjected to a nonlinear curvelet-based primary-multiple separation scheme, either using the total multiples, without any division in categories, and with a cascade of four thresholding processes with thresholds defined in terms of one single category of predicted multiples. The results of this procedure are displayed in Figure 4, which shows a comparison of these two types of primary-multiple separation with the input data including the multiples. Note the extra suppression of diffracted multiples for the case where the four different categories of multiples were used in a cascaded process (Figure 4c) as opposed to the one-term result (Figure 4b). The arrows point at the part with the strongest 3D effects. During this cascaded process, the thresholds can be controlled for each individual multiple contribution which led to the improved result.

\section{Conclusions}

In this paper a strategy is described that may help to extend the 2D SRME methodology in the case of 3D effects. As the diffractive part of the input data is expected to exhibit the most 3D effects, it is proposed to use the methodology to separate data into a reflective and a diffractive part. As multiple prediction is described by a convolution between an operator dataset and the original data, and each of those datasets can be split into two parts, this renders four categories of predicted multiples. In the subtraction phase, a multi-panel curvelet-based primary-multiple separation process can be utilized, which led to a better multiple suppression result compared with a similar process using all multiples in one step for a 2D Gulf of Mexico dataset. Thus, with this increased flexibility $3 \mathrm{D}$ effects in the data can be better accommodated.

\section{Acknowledgments}

The author thanks WesternGeco for providing the Gulf of Mexico, Mississippi Canyon dataset. We also would like to thank the authors of CurveLab for making their codes available. The examples were prepared with Delphi, Seismic Unix and Madagascar (rsf.sourceforge.net).

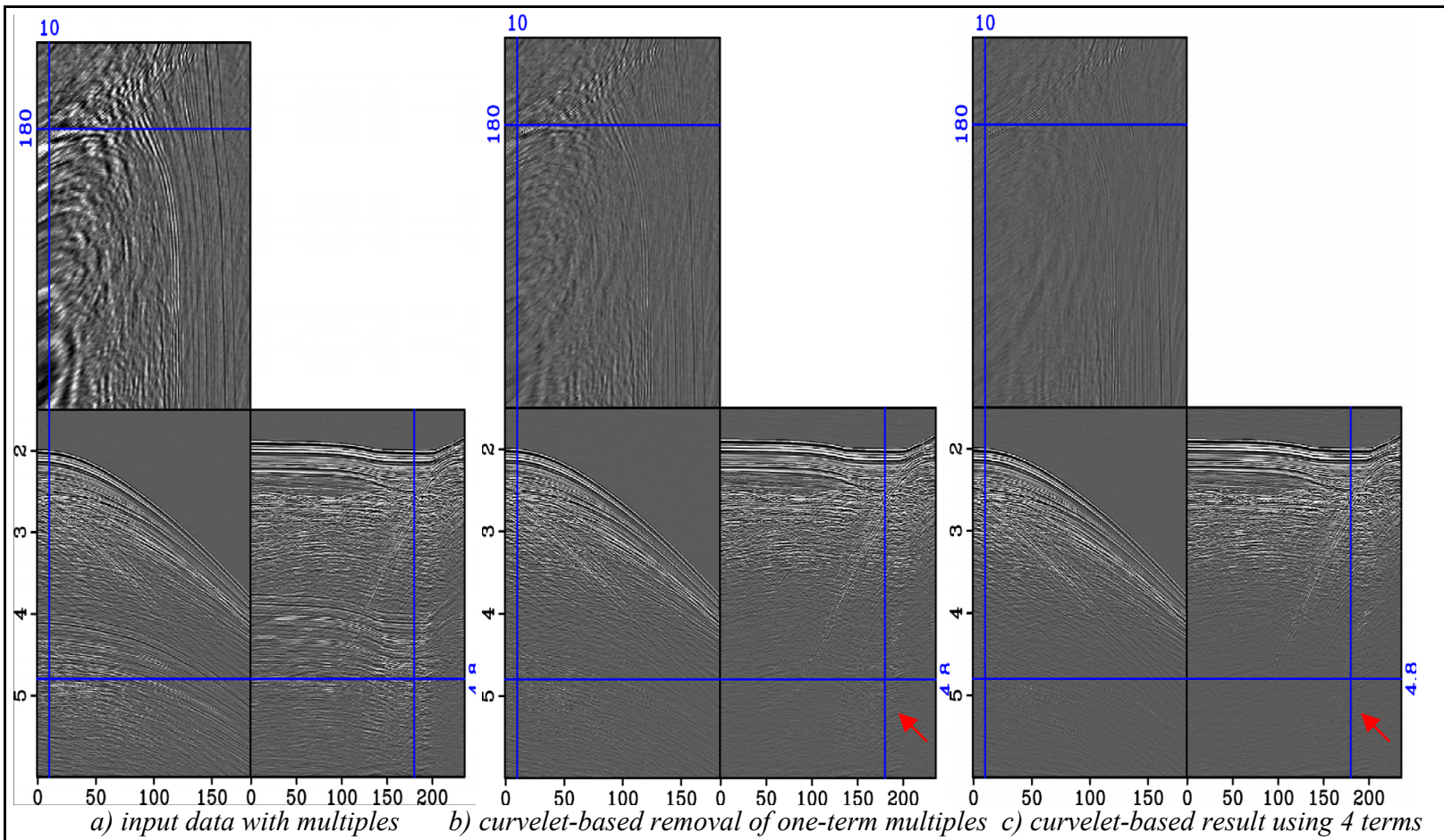

Figure 4: Example of curvelet-based removal of multiples using either all predicted multiples in one thresholding process (b) versus the result of using each of the four categories of predicted multipels in a separate thresholding procedure. Note the improved suppression of the diffracted multiples in the latter case (e.g. at the arrows). Also observe the cleaner appearance of the time slice in (c). 